\title{
The Future of Pulsar Timing Arrays
}

\author{
B. W. Stappers \\ Jodrell Bank Centre for Astrophysics, School of Physics and Astronomy, The University of \\ Manchester, Manchester M13 9PL, UK \\ email: ben.stappers@manchester.ac.uk
}

\begin{abstract}
Significant advances have been made in the sensitivity of pulsar timing arrays for the detection of gravitational waves in the last decade. This presentation looked forward to consider where the development of pulsar timing arrays might go as we head towards the Square Kilometre Array (SKA) and then beyond. I reviewed where progress needs to be made in terms of sensitivity to gravitational waves, including improvements to existing observing approaches and new telescopes such as MeerKAT and FAST and techniques like LEAP. The dramatic increase in the number of millisecond pulsars is presented and how that might affect progress towards a first detection is discussed. Developments in analytic techniques were also discussed, including the removal of interstellar medium effects, red noise and pulse profile variations. A summary of how the SKA can contribute through an increased millisecond pulsar population and pulsar timing sensitivity was presented. With the likelihood that the SKA will implement some form of Key Science Project approach, some ideas of how will this affect how the International Pulsar Timing Array effort and how it might evolve into a KSP were discussed.
\end{abstract}

Keywords. pulsars, gravitational waves, radio astronomy

\section{Introduction}

A pulsar timing array (PTA) aims to use precision timing measurements of the arrival times of pulsars to make a direct detection of gravitational waves (e.g., Romani 1989, Foster \& Backer 1990). To achieve the requisite timing precision millisecond pulsars are used and to achieve sensitivity to the expected stochastic background signal an array of pulsars distributed across the sky is required (Hellings \& Downs 1983). This background may be the result of events during the formation of the Universe, the evolution of cosmic strings and binary supermassive blackholes. As well as a stochastic background it may be possible that we are able to detect gravitational waves from individual sources. Both of these possibilities are discussed in more detail elsewhere in these proceedings.

Although proposed more than three decades ago, it is really only in the last decade or so that significant progress using an array of pulsars has really developed. There are now three main observational efforts around the globe to collect pulsar timing data to undertake the search for gravitational waves: the Parkes Pulsar Timing Array (PPTA, Manchester et al. 2013); the European PTA (EPTA, Desvignes et al. 2015); and the North American Nanohertz Observatory for Gravitational Waves (NANOGrav, Arzoumanian al. 2015). In 2008, it was recognised that by combining these efforts they could be more than the sum of their parts. Following a meeting in Leiden in 2010, the basis for the current structure of the International Pulsar Timing Array $\dagger$ was established. The IPTA is currently a consortium of consortia with membership of one of the founding PTAs, listed above, leading to membership. While originally built around the associated observatories, the PTAs have broadened considerably in scope to include many experts in areas of theory, instrumentation, and simulation. As will be discussed later in this proceedings,

$\dagger$ http://www.ipta4gw.org/ 
this model may need some revision as the search for gravitational waves encompasses more telescopes and countries.

\section{The IPTA and the first data release}

One of the main aims of the IPTA is to facilitate the easy sharing of data between the different PTAs (Verbiest et al. 2015). With this in mind the first project established was to generate a first data release which would combine the data for eight telescopes for 49 millisecond pulsars. Depending on the pulsar, this data set spanned 5-21 years and up to 5 frequencies. It is fair to say that the scope of this challenge was underestimated with the sheer volume of data, the large number of observing systems (many more than the number of telescopes) and different analysis procedures. A detailed discussion of these issues and improved approaches for future data combinations are presented in Verbiest et al. (2015). Despite these difficulties the combination of these data sets are a valuable resource for current and future analysis development. They show how the multi-telescope data allow the separation of systematic and frequency band dependent effects with much greater efficacy than in the individual data sets from the PTAs.

As we look forward to the next data releases what can we expect? There will be longer time baselines for all the pulsars, and in particular for those discovered more recently. The EPTA implemented wide-bandwidth backends more than five years ago which will provide significantly improved time resolution and sensitivity. The NANOGrav project has expanded its list of pulsars significantly and there will be improved multi-frequency coverage. Last but not least there will be improved data reduction techniques built on the experiences of the first data combination.

\section{The push for ever greater sensitivity with current telescopes}

How do we proceed towards a detection? In 2013 a paper by Siemens et al. (2013) established some scaling relations which pointed the way forward. They discussed two regimes related to the weak and intermediate significance of detection. In the former the significance scales as $N C A^{2} T^{13 / 3} / \sigma^{2}$ while in the latter it scales as $N C^{3 / 26}(A / \sigma)^{3 / 13} T^{1 / 2}$, where $N$ is the number of pulsars in the array, $C$ the cadence, $A$ the expected GW background amplitude, $T$ is the length of the pulsar timing data set and $\sigma$ the rootmean-square of the timing residuals. We are currently in the weak regime and so the important parameters are $T$ and $\sigma$, where the latter is directly linked to the timing precision but also requires that we remove or reduce any systematics. Thus, to improve $\sigma$ we need to look to improving the sensitivity of our observations but also mitigation of the influence of the interstellar medium and looking to new timing methodologies. While these factors remain important in the intermediate significance regime, it becomes more important to increase the number of pulsars which can be included in the array and so it requires that we find as many suitable millisecond pulsars as possible.

To improve $\sigma$ with the current telescopes, once they are all performing coherent dedispersion, the only recourse is to improve the sensitivity through improving the receivers. This either means lowering the system temperature or increasing the bandwidth. In recent years we have seen the bandwidths increase significantly, typically up to fractional bandwidths of a third to a half of the central frequency. However we are now entering the era of the so-called ultra-wide band feeds which can span up to a couple of octaves. At the Effelsberg telescope they have been commissioning a 1-3 GHz receiver and are also implementing a 4-9 GHz receiver, in both cases the entire band will be sampled and coherently dedispersed. A related project at the Parkes telescope is building a receiver 
and back end system that will use the entire frequency range from $0.7-4 \mathrm{GHz}$ and at the GBT a proposal has been submitted to build a similar system. These receivers have two advantages over current systems, they achieve improvement in sensitivity through the use of wider bands and the simultaneous recording of these wide bands helps to mitigate the influence of the changing dispersion measure which is known to limit the timing of some MSPs. However, they do also come with some issues, the wider bands mean that they span regions of bright radio-frequency interference and so robustness to these signals put demands on the receiver and the backend linearity. Also, we know that the pulse profiles of pulsars vary as a function of frequency, and even if these changes are more subtle for millisecond pulsars, it has been demonstrated that they can adversely affect timing if not taking into account, especially when combined with effects like scintillation (Demorest, et al. 2013). However some new approaches to timing may be able to take this into account (Pennucci et al. 2014, Liu et al. 2014).

The GMRT telescope in India, while not going for the very wideband feeds, is significantly broadening all of its frequency bands and improving the system temperature. When combined with the very large collecting area it will be an extremely useful addition to the PTA telescopes. Another method to improve sensitivity has been explored in the Large European Array for Pulsars project (Bassa, et al. 2015). LEAP combines the signals from the five largest telescopes in Europe: the Effelsberg telescope, the Lovell telescope, the Nançay radio telescope, the Sardinia Radio Telescope and the Westerbork Synthesis Radio Telescope. When combined coherently these data give the equivalent sensitivity of a dish with a 200-m diameter, making it the largest steerable dish for precision timing. The project has already collected more than three years of data and is providing extremely precise times of arrival and is looking to expand in both bandwidth and number of telescopes.

These improvements and innovations address the issue of sensitivity mainly, the cadence itself is already pretty high with many of the pulsars observed being common to all PTAs and thus can be observed as often as once every 3 days or so (Verbiest et al. 2015). The other key element as we head into the regime of an intermediate detection is the number of pulsars and this will be discussed below.

\section{Tackling the Interstellar Medium}

As discussed above the wider bandwidths provided by the ultra-broad band receivers will help with our modelling and understanding of the interstellar medium although it does come with its own challenges. The addition of the low frequency arrays with wide bandwidths, like LOFAR, MWA, and LWA are also providing us with valuable views of the interstellar medium and how it changes along any given line of sight. LOFAR has detected more than 50 millisecond pulsars, which is more than $2 / 3$ of those observed, in the frequency range 110-188 MHz (Kondratiev et al. 2015). The low frequencies and large fractional bandwidths mean that these observations are able to track changes in the dispersion measure to values of 1 part in 10000 and better. With good cadence these instruments will therefore allow us to probe the changes on useful timescales. How these exactly translate to changes seen at higher frequencies still needs to be ascertained (Cordes et al. 2015, Hassall et al. 2012).

\section{New and upgraded telescopes on the horizon}

As well as improvements to telescopes discussed above there are some new telescopes on the horizon: CHIME, MeerKAT and FAST, as well as a major upgrade for the 
Molongolo telescope, called UTMOST and restarting of pulsar timing with the Deep Space Network dishes. CHIME and MOST are wide field-of-view instruments which will be able to get high cadence observations of many millisecond pulsars. Even if these data are not as sensitive as the some other telescopes the sheer volume of observations will enable monitoring of events such as dispersion measure variations and any other shorter timescale noise effects. FAST (China) and MeerKAT (South Africa) will be the most sensitive telescopes for precision pulsars timing in the Northern and Southern Hemisphere respectively. The initial receiver on FAST will be an ultra-wide band system and the combination will be sensitive enough, that for many pulsars the timing precision will be dominated by pulse-phase jitter. MeerKAT will be able to see the entire Southern sky and up to about 50 degrees North. It will be a few times more sensitive than the Parkes telescope and will use $750-1500 \mathrm{MHz}$ wide bands in 3 frequency ranges from about 500 $\mathrm{MHz}$ to $3.0 \mathrm{GHz}$. It will also be able to form sub-arrays of dishes, so this will be useful for observations of jitter dominated pulsars.

\section{More pulsars for PTAs}

Once we get into the regime of a low significance detection of gravitational waves from a stochastic background the number of pulsars in the pulsar timing array becomes more and more important. To this end there are many pulsar surveys being undertaken and planned. To capture the flavour of this, i.e., the list may not be exhaustive, there are pulsar surveys currently being undertaken with the following telescopes: Parkes, Effelsberg, LOFAR, Arecibo, GBT, and the GMRT. These surveys also span a frequency range from $150 \mathrm{MHz}$ to $1400 \mathrm{MHz}$. As well as these blind surveys, the radio follow up of unidentified gamma-ray sources discovered with Fermi has proved to be extremely effective at revealing the location of millisecond pulsars (e.g., Ray et al. 2012). All these searches have led to more than a doubling of the millisecond pulsar population in the last five years (see also McLaughlin these proceedings).

Surveys with the next generation telescopes, FAST and MeerKAT will further enhance the population of PTA suitable pulsars. FAST will survey the sky initially with the wide band feed mentioned above and then with a 19-beam system working at $1400 \mathrm{MHz}$ and it is estimated that it will find at least 200 new millisecond pulsars. Once outfitted with the proposed beamformer capable of 400 beams, MeerKAT will also be able to complete blind surveys with the best sensitivity ever achieved from the Southern Hemisphere, even before then it will perform deep searches of the still unidentified Fermi sources.

\section{Looking forward to the SKA}

In all three major areas of improving our sensitivity to gravitational waves the SKA will be revolutionary. A more detailed discussion of the role of the SKA in pulsar research is discussed in the SKA science book: see Kramer \& Stappers (2015) for an overview and the chapters on the pulsar census (Keane et al. 2015) and gravitational wave astronomy (Janssen et al. 2015) specifically to this topic.

Pulsar searches with the SKA will be undertaken with both SKA1-LOW and SKA1-MID. The former will be used to search most of the Southern sky excluding the Galactic plane, seen from Australia, at frequencies around $300 \mathrm{MHz}$ and the latter will be used to search the Galactic plane, $|b|<5^{\circ}$, at frequencies around $1500 \mathrm{MHz}$ and the outer plane, $|b|<10^{\circ}$ at frequencies near $800 \mathrm{MHz}$. The Northern sky not accessible to SKA1-LOW will also be observed at the intermediate frequency. This combined approach to the pulsar survey results in the optimal survey return for the SKA where the total sample of 
millisecond pulsars estimated to be discovered is more than 1500. Looking further forward the projections for a combined LOW and MID frequency search with the full SKA will result in the discovery of at least 4000 millisecond pulsars.

As well as this transformational step in the number of available millisecond pulsars for inclusion in pulsar timing arrays the enormous increase in sensitivity will dramatically improve the precision of pulsar timing and the number of millisecond pulsars that can be timed to a precision that makes them suitable for inclusion. The high precision timing will most likely be done in Bands $2(0.9-1.6 \mathrm{GHz})$ and $3(1.6-3.0 \mathrm{GHz})$ of SKA-MID, however Bands $1(0.35-1.0 \mathrm{GHz})$ \& 2 \& 5 (>10 GHz) will be delivered first and so these will be used initially. The lower bands are essential for ISM effects and the higher bands will be used for Galactic centre regions. Sub-arraying of the dishes will be used for stronger pulsars that are dominated by jitter noise and will also provide flexibility and efficiency of observing. Pulsar timing with SKA1-LOW will be useful for interstellar weather measurements and correction. Overall the SKA will need to support regularcadence observations, at multiple frequencies over a long timespan of a large number of pulsars. It is also important to note that in the future VLBI with/and including the SKA will be able to provide independent astrometry that will assist in achieving immediate high-precision timing and also providing increased sensitivity for $f=1 \mathrm{yr}^{-1}$ and $2 \mathrm{yr}^{-1}$ frequencies of gravitational waves.

\section{Studying gravitational waves with the SKA}

We can envisage two different scenarios in the SKA era for using pulsar timing arrays to try to directly detect gravitational waves at nano-hertz frequencies: (i) they haven't yet been detected and (ii) they have been detected. In the first scenario the SKA will provide the necessary improvements in sensitivity and number of pulsars to enable a detection or the limits obtained for gravitational wave emission will become so stringent that they will require a complete rethink of the galaxy formation models. For much more detail on the SKA and the gravitational wave detection capabilities see Rosdao et al. (2015).

In the second scenario when a detection has already been made then of course the first role of the SKA will be to confirm the detection of the signal. After that, independent of whether the initial detection was made with the SKA or not, it will be necessary to characterise the spectrum of the source. In the case of a stochastic background this will enable the identification of the source of the gravitational waves, i.e., whether it is merging supermassive blackholes or cosmic strings. It will also be important to undertake searches for any anisotropy in the background and/or identify any single sources. The SKA will also be able to begin to contribute to the era of gravitational wave astronomy. This will include being able to probe the merger history of binary supermassive blackholes and explore constraints on galaxy formation models. As shown by Lee et al. (2008) it will also be possible to test the properties of gravity with the SKA by studying the polarisations of the gravitational waves, depending on the nature of the polarisation this will result in different Hellings and Downs correlations. Also, if the graviton has mass, this too will result in modifications of the correlation, although these will require large numbers of pulsars to be able to identify any deviations (Lee et al. 2010). It should also be possible to quite precisely locate single sources of gravitational waves with the SKA (Lee et al. 2011).

\section{Conclusions}

It is clear that we are at a cross roads in the development of the IPTA. The current construction based on a consortium of consortia has worked until now with new people 
encouraged to join one of the three existing PTAs which make up the consortia. However, as we move forward there are considerable new telescope resources coming online, like FAST, upgraded GMRT and MeerKAT which will likely not want to come under the banners of the existing PTAs. Also, many of these new telescopes and projects involve multiple members from the existing PTAs, so the distinctions are becoming blurred. How to reformat the IPTA is currently a matter of debate in the IPTA steering committee and the community is encouraged to contribute.

Thrown into this discussion is the situation of the SKA. At present the SKA is investigating the size, scope and number of Key Science Projects and the idea of searching for gravitational waves with a pulsar timing array has been a long held aim of the SKA and it is likely to be a KSP or part of a KSP. At the time when such a KSP is proposed for it will be important to understand how it fits with the existing or future IPTA structure.

The future for gravitational wave research with PTAs is looking very bright. The prolific increase in the number of new pulsars and improvements to current instrumentation, and the new instruments and telescopes on the near horizon make detection in the near future ever more likely. The IPTA data sets from these instruments are extremely valuable, but they need to be generated on a reasonable timescale which requires a strong commitment from the individual PTAs. On the near horizon, new techniques like LEAP, and the new telescopes FAST and MeerKAT will give us a much needed sensitivity boost. Whether or not an initial detection is already made, the SKA will herald an era of gravitational wave astronomy using PTAs.

\section{Acknowledgement}

I would like to thank members of all of the PTAs for contributions for the information presented in these slides, in particular Michael Kramer, Dick Manchester, Joris Verbiest and Scott Ransom.

\section{References}

Arzoumanian, Z., et al. The NANOGrav Collaboration 2015, ApJ, in press; arXiv:1505.07540 Bassa, C., et al. 2015, MNRAS, submitted

Cordes, J. M., Shannon, R. M., \& Stinebring, D. R. 2015, ApJ, submitted; arXiv:1503.08491

Demorest, P. B., et al. 2013, ApJ, 762, 94

Desvignes, G., et al. 2015, MNRAS, submitted

Foster, R. S. \& Backer, D. C. 1990, ApJ, 361, 300

Hassall, T. E., et al. 2012, A\&SA, 543, A66

Hellings, R. W. \& Downs, G. S. 1983, ApJ, 265, L39

Janssen, G., Hobbs, G., McLaughlin, M., et al. 2015, "Gravitational Wave Astronomy with the SKA," in Advancing Astrophysics with the Square Kilometre Array, Proc. Sci., eds. T. L. Bourke, et al., id 37

Keane, E., Bhattacharyya, B., Kramer, M., et al. 2015, "A Cosmic Census of Radio Pulsars with the SKA," in Advancing Astrophysics with the Square Kilometre Array, Proc. Sci., eds. T. L. Bourke, et al., id 40

Kondratiev, V. I., Verbiest, J. P. W., Hessels, J. W. T., et al. 2015, A\&A, in press; arXiv:1508.02948

Kramer, M. \& Stappers, B. 2015, "Pulsar Science with the SKA," in Advancing Astrophysics with the Square Kilometre Array, Proc. Sci., eds. T. L. Bourke, et al., id 36

Lee, K. J., Jenet, F. A., \& Price, R. H. 2008, ApJ, 685, 1304

Lee, K., Jenet, F. A., Price, R. H., Wex, N., \& Kramer, M. 2010, ApJ, 722, 1589

Lee, K. J., Wex, N., Kramer, M., et al. 2011, MNRAS, 414, 3251

Liu, K., Desvignes, G., Cognard, I., et al. 2014, MNRAS, 443, 3752

Manchester, R. N., Hobbs, G., Bailes, M., et al. 2013, PASA, 30, 17 
Pennucci, T. T., Demorest, P. B., \& Ransom, S. M. 2014, ApJ, 790, 93

Ray, P. S., Abdo, A. A., Parent, D., et al. 2012, 2011 Fermi Symposium proceedingseConf C110509; arXiv:1205.3089

Romani, R. W. 1989, "Timing a millisecond pulsar array," in Timing Neutron Stars, eds. H. Ögelman \& E. P. J. van den Heuvel (Kluwer: New York) p. 113

Rosado, P., Sesana, A., \& Gair, J. 2015, MNRAS, 451, 2417

Siemens, X., Ellis, J., Jenet, F., \& Romano, J. D. 2013, Classical Quant. Grav., 30, 224015

Verbiest, J. P. W., et al. 2015, MNRAS, submitted 